\title{
WEAK $q$-RINGS WITH ZERO SINGULAR IDEAL
}

\author{
SAAD MOHAMED AND SURJEET SINGH ${ }^{1}$
}

\begin{abstract}
A ring $R$ is called a (right) $w q$-ring if every right ideal not isomorphic to $R_{R}$ is quasi-injective. The main result proved is the following: Let $R$ be a ring with zero singular ideal, then $R$ is a wq-ring if and only if either $R$ is a $q$-ring, or $R=\left[\begin{array}{ll}D & D \\ 0 & D\end{array}\right]$ for some division ring $D$, or $R$ is such that every right ideal not isomorphic to $R_{R}$ is completely reducible.
\end{abstract}

Throughout this paper, the rings considered are with unity and every module is a unital right module. A ring $R$ is called a (right) $q$-ring if every right ideal of $R$ is quasi-injective [5]. Such rings and their dual concept have been studied by many authors. Recently Byrd [1] determined the structure of $q$-rings without imposing any finiteness conditions. In [8], the present authors initiated the study of those rings $R$ whose right ideals not isomorphic to $R_{R}$ are quasi-injective; such rings are called weak $q$-rings (in short $w q$-rings). The structure of $w q$-rings under some finiteness conditions was determined in [8]. In this paper we study $w q$-rings with zero right singular ideal, which need not satisfy any finiteness conditions. A characterization of such rings is given in Theorem (2.9). The structures of the right socle and the Jacobson radical of these rings are also determined.

1. Preliminaries. For definition and some properties of quasi-injective modules, we refer the reader to Johnson and Wong [6] (see also Faith [2]). For any module $M_{R}$, the smallest cardinal $\alpha$ such that any direct sum in $M_{R}$ has at most $\alpha$ components is called the dimension of $M$ (denoted by $d(M)$ ). A submodule $N$ of a module $M$ is called a complement submodule if $N$ is a complement of some submodule $K$ of $M$. The following two results are due to Miyashita [7]:

(1.1) Theorem. Any complement submodule of a quasi-injective module $M$ is a summand of $M$.

(1.2) THEOREM. A finite dimensional quasi-injective module is a direct sum of uniform modules.

Received by the editors August 29, 1978.

AMS (MOS) subject classifications (1970). Primary 16A52; Secondary 16A08, 16A66.

Key words and phrases. Quasi-injective module, singular submodule, dimension of a module, hereditary ring, $q$-ring.

${ }^{1}$ This research was done while Surjeet Singh was visiting Kuwait University in July-August, 1978. 
Ivanov [4] proved the following:

(1.3) Lemma. Let $A$ and $B$ be right ideals of a q-ring $R$. If $A \cap B=0$ then for any $R$-homomorphism $f: A \rightarrow B, f(A)$ is semisimple (i.e., completely reducible).

On similar lines, the following can be proved:

(1.4) LEMMA. Let $A$ and $B$ be any modules such that for every essential submodule $C$ of $B, A \oplus C$ is quasi-injective. Then for any homomorphism $f$ : $A \rightarrow B, f(A)$ is completely reducible.

The following is well known:

(1.5) Lemma. Let $A$ and $B$ be any modules. If $A \oplus B$ is quasi-injective, then any monomorphism from $A$ to $B$ splits.

For any module $M_{R}, Z(M)$ and $\hat{M}$ will denote the singular submodule and the injective hull of $M$, respectively. It is well known that if a ring $R$ has $Z(R)=0$, then $\hat{R}$ is a right self-injective ring of which $R$ is a subring (see [2]).

For any right ideal $A$ of a ring $R$ with $Z(R)=0$, let

$$
A^{*}=\{x \in R: \hat{R} x \subset A\} \text {. }
$$

Then $A^{*}$ is a right ideal of $R$ contained in $A$. It is proved in [8, Lemma (1.1)] that $A^{*}$ is a left ideal in $\hat{R}$ and is a quasi-injective $R$-module; if in addition $A$ is essential in $R_{R}$, then $A=A^{*}$ if and only if $A$ is quasi-injective.

2. Rings with zero singular ideal. We start by the following general result.

(2.1) Lemma. Let $A$ be a quasi-injective right ideal in a wq-ring $R$. If $A$ contains a right regular element, then $R$ is a q-ring.

Proof. Let $a \in A$ be a right regular element. Then $R \simeq a R \subset A$. This implies that $A$ is injective and $\hat{R}$ is embedded in $A$. Let $R=B \oplus C$ where $B \simeq \hat{R}$. Then $B=B_{1} \oplus B_{2}$ where $B_{1} \simeq B$ and $B_{2} \simeq \hat{C}$. If $B_{2} \oplus C \simeq R$, then $B_{2} \oplus C$ is quasi-injective. Since $C$ embeds in $B_{2}$, then Lemma (1.5) gives that $C$ is injective. Hence $R_{R}$ is injective and $R$ is a $q$-ring. On the other hand, $B_{2} \oplus C \simeq R$ implies that $R \simeq \hat{R} \oplus R$. This in turn implies that $R$ contains an infinite direct sum $\Sigma \oplus R_{i}$ of copies of $R$. Since $\Sigma \oplus R_{i} \simeq R$, we get $\Sigma \oplus R_{i}$ is quasi-injective. Hence $R_{R}$ is injective. This completes the proof.

The following is proved in [8, Theorem (2.7)].

(2.2) THEOREM. Let $R$ be a ring such that $Z(R)=0$. If $R$ is a wq-ring, then either $R$ is a right PID, or $R$ is a strongly regular right self-injective ring (hence a $q$-ring) or $R$ has nonzero right socle.

Since right PID's and $q$-rings are trivial cases of $w q$-rings, we will be mainly interested in those $w q$-rings which are not of these two types. As such, in all the lemmas to follow, $R$ will be a $w q$-ring with $Z(R)=0$ which is neither a right PID nor a $q$-ring. $S$ will denote the right socle of $R$. We note that $R$ has 
no nontrivial central idempotents [8, Lemma (1.5)].

(2.3) LEMMA. $S$ is essential in $R_{R}$.

Proof. By Theorem (2.2), $S \neq 0$. Suppose $S$ is not essential in $R_{R}$. Let $C$ be a complement of $S$. We claim that $S \oplus C \not R$. Suppose not. Then $S=g R$ for some nontrivial idempotent $g$. This implies that every minimal right ideal of $R$ is projective, and hence

$$
g R(1-g)=0=(1-g) R g .
$$

Thus $g$ is a central idempotent, a contradiction. Therefore $S \oplus C \nsim R$. Hence $S \oplus C=(S \oplus C)^{*}$, a left ideal in $\hat{R}$. Let $a R$ be a minimal right ideal of $R$. There exist $x \in \hat{R}$ such that $a x a=a$. Then $f=x a$ is an idempotent in $R$ and $a R \simeq f R$. This proves that every minimal right ideal of $R$ is projective. We proceed to show that $C$ is a left ideal in $\hat{R}$. On the contrary, let $y \in \hat{R}$ be such that $y C \not C$. However $y C \subset S \oplus C$. This defines a nonzero homomorphism $\eta: C \rightarrow S$. Since $\operatorname{Im} \eta$ is projective and completely reducible, we get $S \cap C \neq 0$. This is a contradiction. Hence $\hat{R} C \subset C$. As $\hat{R}$ is a regular ring, $C$ contains a nontrivial idempotent $e$. Then $C=e R \oplus(1-e) C$. Since $\operatorname{Soc}\left(C_{R}\right)$ $=0$, Lemma (1.4) gives

$$
\operatorname{Hom}(e R,(1-e) C)=0=\operatorname{Hom}((1-e) C, e R) .
$$

Consequently,

$$
(1-e) C e=0=e R(1-e) C .
$$

Now, $e R(S \oplus(1-e) C)=e R S+e R(1-e) C=0$. So that

$$
S \oplus(1-e) C \subset(1-e) R \text {. }
$$

Since $S \oplus C \subset^{\prime} R$, we get $S \oplus(1-e) C \subset^{\prime}(1-e) R$. Then $Z(R)=0$ implies $e R(1-e) R=0$. Also $(1-e) R e=(1-e) C e=0$. Hence $e$ is a central idempotent, a contradiction. This completes the proof.

(2.4) COROllaRy. Let $R$ be a wq-ring with $Z(R)=0$ which is neither a right $P I D$ nor a q-ring. Then any minimal right ideal of $R$ is projective and every homogeneous component of $\operatorname{Soc}\left(R_{R}\right)$ contains nonzero idempotents.

(2.5) Proposition. If $R$ is a wq-ring with $Z(R)=0$, then $R / R^{*}$ is a right PID.

Proof. The result is obvious for a right PID or a $q$-ring. So assume that $R$ is not one of these rings. By Lemma (2.3), $S=\operatorname{Soc}\left(R_{R}\right) \subset{ }^{\prime} R_{R}$. Then $S=S^{*}$ $\subset R^{*}$, and hence $R^{*} \subset{ }^{\prime} R_{R}$. Let $A / R^{*}$ be a nonzero right ideal of $R^{*}$. Then $A \supsetneqq R^{*}$ implies that $A \simeq R$. So that $A=a R$ for some right regular element $a$ of $R$. Since $\hat{R}$ is a regular ring, $a$ has a left inverse in $\hat{R}$. Hence $a R^{*}=a R$ $\cap R^{*}$. Now

$$
A / R^{*}=\left(a R+R^{*}\right) / R^{*} \simeq a R /\left(a R \cap R^{*}\right)=a R / a R^{*} \simeq R / R^{*} .
$$

This proves that every nonzero right ideal of $R / R^{*}$ is isomorphic to $R / R^{*}$. Hence $R / R^{*}$ is a right PID. 
(2.6) Proposition. Let $R$ be a wq-ring which is neither a right PID nor a $q$-ring. If $d(R)>2$, then $d(R)=\infty$; in fact, no homogeneous component of $\operatorname{Soc}\left(R_{R}\right)$ is finitely generated.

Proof. Let $H$ be a homogeneous component of $S$. By Corollary (2.4), there exists an idempotent $e \in H$ such that $e R$ is a minimal right ideal. Suppose that $d(H)<\infty$. If $(1-e) R \simeq R$, then $(1-e) H=(1-e) R \cap H \simeq H$. This contradicts $d(H)<\infty$. Hence $(1-e) R \nsim R$, and $(1-e) R$ is quasi-injective. Note that $(1-e) H \neq 0$, since otherwise $H=e R$ and $e$ becomes a central idempotent. Let $B$ be the maximal essential extension of $(1-\mathrm{e}) H$ in $(1-e) R$. Then by Theorem $(1.1),(1-e) R=B \oplus C$ for some right ideal $C$. Then $B$ is quasi-injective and $d(B)<\infty$. By Theorem (1.2),

$$
B=U_{1} \oplus U_{2} \oplus \cdots \oplus U_{t}
$$

for finitely many uniform submodules $U_{i}$. Since $d(R)>2$, eR $\oplus U_{i} \simeq R$, and so $e R \oplus U_{i}$ is quasi-injective. Then as $e R$ is embeddable in $U_{i}, e R \simeq U_{i}$ by Lemma (1.5). This gives $B=(1-e) H$, and so $H$ is a summand of $R$. This is again a contradiction. Hence the result follows.

(2.7) Lemma. If $d(R)>2$ and if $B$ is any right ideal of $R$ such that $B \cap S$ is finitely generated, then $B \subset S$.

Proof. Since $S \subset{ }^{\prime} R_{R}$ by Lemma (2.3), $B \cap S \subset{ }^{\prime} B$. Then $B \cap S$ is finitely generated implies $d(B)<\infty$. However $d(R)=\infty$ by the above proposition. Consequently $B$ is quasi-injective, and by Theorem (1.2), $B=$ $\sum_{i=1}^{t} \oplus U_{i}$, with each $U_{i}$ uniform right ideal. Since each homogeneous component of $S$ has infinite dimension by Proposition (2.6), we can find minimal right ideals $A_{i}$ such that $A_{i} \cap U_{i}=0$ and $A_{i} \simeq \operatorname{Soc}\left(U_{i}\right)$. Then $A_{i} \simeq$ $U_{i}$ by the quasi-injectivity of $A_{i} \oplus U_{i}$. This proves that $B \subset S$.

(2.8) LEMMA. If $d(R)>2$, then any right ideal $A$ of $R$ not isomorphic to $R$, is completely reducible.

Proof. Let $a \in A$. By Lemma (2.1), $a R \not R$ and consequently $a R$ is quasi-injective. We claim that every homogeneous component of $\operatorname{Soc}(a R \cap$ $S$ ) is finitely generated. Suppose not. Then we get a direct sum

$$
\sum_{i=1}^{\infty} \oplus A_{i} \oplus \sum_{i=1}^{\infty} \oplus B_{i}
$$

of mutually isomorphic minimal right ideals $A_{i}$ and $B_{i}$ contained in $a R$. Let $D$ be a complement submodule of $\Sigma \oplus A_{i}$ in $a R$ containing $\Sigma \oplus B_{i}$. Then by Theorem (1.1), $D$ is a summand of $a R$. Now $\Sigma \oplus A_{i} \oplus D$ is quasi-injective and $\Sigma \oplus A_{i}$ is embeddable in $D$; so by Lemma (1.5), $\Sigma \bigoplus A_{i}$ is isomorphic to a summand of $D$. Consequently $\Sigma \oplus A_{i}$ is finitely generated; a contradiction. Hence every homogeneous component of $\operatorname{Soc}(a R \cap S)$ is finitely generated.

Now assume that $a R$ is not completely reducible. Then by Lemma (2.7), 
$d(a R \cap S)=\infty$. Hence $\operatorname{Soc}(a R \cap S)$ contains infinitely many homogeneous components. Since no homogeneous component of $S$ is finitely generated by Proposition (2.6), we can find an infinite direct sum $\Sigma \oplus C_{i}$ of minimal right ideals such that $\left(\Sigma \oplus C_{i}\right) \cap a R=0$ and $\Sigma \oplus C_{i}$ embeds in $a R$. However, this is a contradiction, since $a R \oplus \Sigma \oplus C_{i}$ is quasi-injective. Therefore $a R$ is completely reducible. This completes the proof.

We now prove the main theorem.

(2.9) THEOREM. Let $R$ be a ring with $Z(R)=0$. Then $R$ is a wq-ring if and only if:

(1) $R$ is a q-ring, or

(2) $R=\left[\begin{array}{ll}D & D \\ 0 & D\end{array}\right]$ for some division ring $D$, or

(3) Every right ideal of $R$, not isomorphic to $R$ is completely reducible.

Proof. Let $R$ be a $w q$-ring. Suppose $R$ is not a $q$-ring. If $d(R)$ is finite, then by [8, Theorem (2.4)], $R$ is of type (2), or $R$ is a right PID and as such is of type (3). If $d(R)$ is infinite, then by the above Lemma, $R$ is of type (3). This proves the necessity. The sufficiency is obvious.

The following is a consequence of the above theorem and Corollary (2.4).

(2.10) THEOREM. If $R$ is a wq-ring with $Z(R)=0$ and if $R$ is not a q-ring, then $R$ is right hereditary.

The next proposition gives some information about the socle and the Jacobson radical of $w q$-ring $R$ with $Z(R)=0$.

(2.11) Proposition. Let $R$ be a wq-ring with $Z(R)=0$ and let $J$ be its Jacobson radical. Then $J^{2}=0$. Further if $R$ is not a q-ring and if $d(R)>2$, then every homogeneous component of $\operatorname{Soc}\left(R_{R}\right)$ contains an infinite number of orthogonal idempotents.

Proof. It is obvious from [8, Theorem (2.4)] that if $R$ is a $q$-ring or $d(R) \leqslant 2$, then $J^{2}=0$. So we assume that $R$ is not a $q$-ring and $d(R)>2$. Let $A=J \cap S$, then $A^{2}=0$. Write $S=A \oplus B$ for some right ideal $B$ of $R$. Then $B$ is a sum of minimal right ideals each of which is generated by an idempotent. Further by Corollary (2.4), every homogeneous component of $S$ has nonzero intersection with $B$. Let $H$ be a homogeneous component of $B$. If $H$ is finitely generated, then $H=e R$ for some idempoent $e \in R$. Then $(1-e) R$ is not completely reducible. It follows by Theorem (2.9) that $(1-e) R \simeq R$. Consequently $(1-e) R=C \oplus D$ with $C \simeq H=e R$ and $D$ $\simeq(1-e) R$. Then

$$
R=e R \oplus C \oplus D .
$$

Since $S J=0, J=D J$ and hence $(e R \oplus C) \cap J=0$. Now

$$
S=e R \oplus C \oplus(D \cap S)=B \oplus A \text {. }
$$

This defines a monomorphism $\theta: e R \oplus C \rightarrow B$. It is clear that $\operatorname{Im} \theta \subset H$ and $d(e R \oplus C)=2 d(H)$. This is a contradiction. Hence every homogeneous 
component of $B$ contains an infinite direct sum. Then $J \oplus B \nsim R$, and by Lemma (2.8), $J \subset S$. Hence $J=A$ and $J^{2}=0$. This completes the proof.

Harada [3] proved that if for two modules $M_{1}$ and $M_{2}, M_{1} \oplus M_{2}$ is quasi-injective, then $M_{1} \simeq M_{2}$ if and only if $\hat{M}_{1} \simeq \hat{M}_{2}$. Using this and Theorem (2.9), one can easily prove the following:

(2.12) Proposition. Let $R$ be a wq-ring with $Z(R)=0$. Then $\hat{R}$ is a prime ring if and only if socle $\left(R_{R}\right)$ is homogeneous.

We end this paper with the following remarks.

(1) We are not aware of an example of a ring $R$ with $Z(R)=0$, in which every right ideal not isomorphic to $R$ is completely reducible, but the ring itself is neither a right PID nor semisimple artinian.

(2) Since a semiprime $w q$-ring has zero right singular ideal [8, Proposition (1.8)], the results on semiprime, and in particular prime, $w q$-rings proved in [8], are immediate consequences of results established above.

\section{REFERENCES}

1. K. A. Byrd, Right self-injective rings whose essential right ideals are two sided, Pacific J. Math. (to appear).

2. C. Faith, Algebra. II, Springer-Verlag, New York, 1975.

3. M. Harada, Note on quasi-injective modules, Osaka Math. J. 2 (1965), 351-356.

4. G. Ivanov, Non-local rings whose ideals are all quasi-injective, Bull. Austral. Math. Soc. 6 (1972), 45-52.

5. S. K. Jain, S. H. Mohamed and S. Singh, Rings in which every right ideal is quasi-injective, Pacific J. Math. 31 (1969), 73-79.

6. R. E. Johnson and E. T. Wong, Quasi-injective modules and irreducible rings, J. London Math. Soc. 36 (1961), 260-268.

7. Y. Miyashita, On quasi-injective modules, J. Fac. Sci. Hokkaido Univ. 18 (1965), 158-187.

8. S. Mohamed and S. Singh, Weak q-rings, Canad. J. Math. 29 (1977), 687-695.

Department of Mathematics, Kuwait University, KuWait

Department of Mathematics, Guru Nanak Dev University, Amritsar, India 